EPJ Web of Conferences 41, 05011 (2013)

DOI: $10.1051 /$ epjconf/20134105011

(C) Owned by the authors, published by EDP Sciences, 2013

\title{
Effect of bridge on energy transfer and photoinduced charge separation in perylene-diimide-naphthalene-bisimide- hexathiophene based donor-bridge-acceptor triads
}

\author{
J. Zaks ${ }^{1,4}$, J. Sun ${ }^{2,3}$, S. Kirmayer ${ }^{3,5}$, J. J. Urban ${ }^{5}$, T. D. Tilley ${ }^{2}$, R. A. Segalman, ${ }^{1,3,5}$ G. R. Fleming ${ }^{1,2,4}$ \\ ${ }^{1}$ Applied Science and Technology Graduate Group, University of California, Berkeley, Berkeley, \\ California, 94720, USA \\ ${ }^{2}$ Department of Chemistry, University of California, Berkeley, Berkeley, California 94720, USA \\ ${ }^{3}$ Department of Chemical and Biomolecular Engineering, University of California, Berkeley, \\ Berkeley, California 94720, USA \\ ${ }^{4}$ Physical Biosciences Division, Lawrence Berkeley National Laboratory, 1 Cyclotron Road, \\ Berkeley, California 94720, USA \\ ${ }^{5}$ The Molecular Foundry, Material Science Division, Lawrence Berkeley National Laboratory, \\ Berkeley, California, 94720, USA
}

\begin{abstract}
Femtosecond transient absorption spectroscopy is performed to assess bridge effects on energy transfer and charge separation in molecular junctions. A short, conjugated bridge can facilitate charge separation from both donor and acceptor, whereas in longer bridges charge separation only occurs from the excited donor.
\end{abstract}

\section{Introduction}

Controlling charge separation on an intramolecular scale in molecular p-n junctions will be important for increasing the efficiency of solar cell devices [1,2]. The effect of the connection between electron donor and electron acceptor materials on the efficiency and yield of charge separation and charge recombination is still a topic of ongoing investigation [3]. To assess the effect of conjugation, length, and rigidity of the bridge between donor and acceptor on charge separation and recombination, we conducted transient absorption (TA) measurements on molecular PN junctions consisting of a hexathiophene (6T) electron donor and a naphthalene bisimide (NDI)perylene bisimide (PDI) dimer electron acceptor.

\section{Experimental Methods}

TA experiments were conducted with a tunable pump and a broadband probe. The source for the 525 nm pump (to excite the PDI acceptor) was an optical parametric amplifier (Coherent OPA 9040) pumped by a $250-\mathrm{kHz}$ Ti:Sapphire regenerative amplifier (Coherent RegA 9050). The amplified output was frequency-doubled to produce the $400 \mathrm{~nm}$ pump (to excite the $6 \mathrm{~T}$ donor). The probe was supercontinuum generated in a $2 \mathrm{~mm}$ sapphire crystal. The probe was frequency-resolved with a spectrometer and the TA was collected with a lock-in amplifier (Stanford Research Systems). The molecules consisted of a $6 \mathrm{~T}$ donor coupled to a PDI-NDI acceptor via three different bridges: a

This is an Open Access article distributed under the terms of the Creative Commons Attribution License 2.0, which permits unrestricted use, distribution, and reproduction in any medium, provided the original work is properly cited. 
short, conjugated bridge (PNC), a longer non-conjugated bridge (PNCC), a flexible, non-conjugated bridge (PNF), and a rigid, non-conjugated bridge (PNR). The molecular structure and absorption and emission spectra of the donor and acceptor molecules are shown in Figs. 1a and 1b. For TA measurements, molecules were dissolved in toluene to an OD of $>0.5$ in a $0.5 \mathrm{~mm}$ cuvette and flowed by a peristaltic pump at room temperature. The energy of the pump was 5-10 nJ/pulse, well below the damage threshold for similar molecules [5].

\section{Results and Discussion}

The TA of all three molecules exhibited positive features corresponding to excited state absorption (ESA) in the 650-960 $\mathrm{nm}$ region and negative features corresponding to ground state bleach (GSB)/stimulated emission (SE) with peaks at 540 and $580 \mathrm{~nm}$. Immediately following excitation of the hexathiophene (6T) at $400 \mathrm{~nm}$, all three molecules exhibited an ESA in the range between 720 and $960 \mathrm{~nm}$, which we ascribe to the ESA of 6T [8]. This feature decayed at a comparable rate to the appearance of GSB/SE signals at $540 \mathrm{~nm}$ (PDI GSB and SE) and $580 \mathrm{~nm}$ (only PDI SE), indicating energy transfer from 6T to PDI (Fig. 1c). The rate of energy transfer was fastest in PNC (700 fs), slower in PNF (1.3 ps) and PNCC (1.4 ps), and slowest in PNR (3 ps).
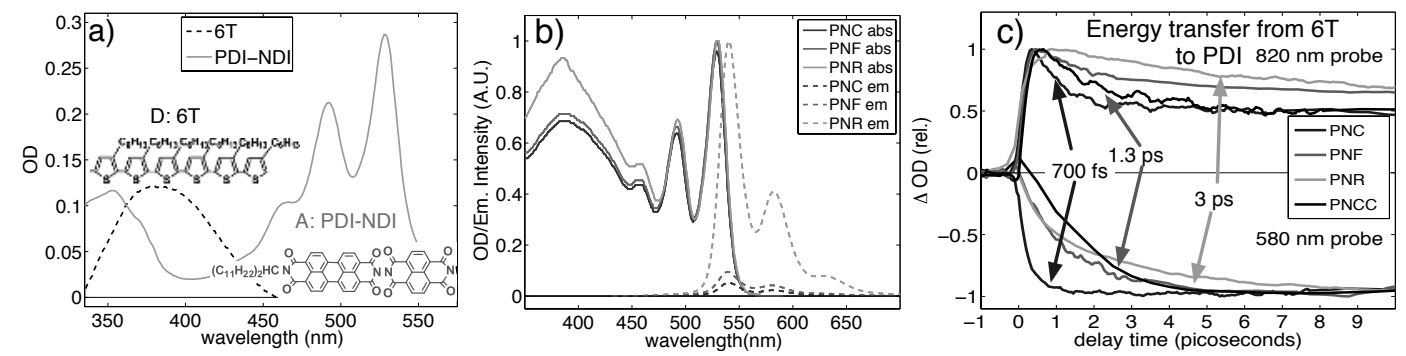

Fig. 1. a) Molecular structure and absorption spectra of donor (6T), acceptor (PDI-NDI) b) Absorption and photoluminescence spectra of molecules. c) TA of molecules excited at $400 \mathrm{~nm}$ and probed $820 \mathrm{~nm}$ and $580 \mathrm{~nm}$, revealing timescale of energy transfer from 6T to PDI.

PNR, PNF, and PNCC exhibited a long-lived feature that absorbed at $720 \mathrm{~nm}$ (Fig. 2a-c). Features that absorb at $720 \mathrm{~nm}$ include the PDI radical anion [4-7] and the 6T triplet, which has an absorption maximum at $680 \mathrm{~nm}[8,9]$. In PNR we observed that GSB of the PDI ground state at 540 $\mathrm{nm}$ persisted for longer than SE from the PDI excited state at $580 \mathrm{~nm}$ (shown in Fig. 2a), indicating that the relaxation of PDI involves a state other than the excited state. It is unlikely that this state is localized on the PDI alone because PDI excited directly at $525 \mathrm{~nm}$ did not form a long-lived state (Fig. 2a, dashed line). Therefore, the long-lived state in PNR is likely to be a charge-separated (CS) state between the 6T and PDI. In PNF (Fig. 2b), the GSB/SE of the PDI at $540 \mathrm{~nm} / 580 \mathrm{~nm}$ decayed with a lifetime of $1.7 \mathrm{~ns} / 1 \mathrm{~ns}$, and the ESA at 720 and 820 decays with a timescale of $\sim 3.6 \mathrm{~ns}$, suggesting that the longer-lived state absorbing at $720 \mathrm{~nm}$ does not entirely involve the PDI moiety, and could be the 6T triplet state [9]. PNCC (Fig. 2c) transient absorption was most similar to that of PNR, except that features at $720 \mathrm{~nm}$ and $820 \mathrm{~nm}$ exhibited a slow rise, suggesting that recombination from the CS state to the ground state is slowest in PNCC.

In contrast to the longer molecules, the formation of a transient species absorbing at $720 \mathrm{~nm}$ appeared in PNC following excitation of both $6 \mathrm{~T}$ at $400 \mathrm{~nm}$ and PDI at $525 \mathrm{~nm}$, as shown in Fig. $2 \mathrm{~d}$. This suggests that a charge-separated state can form from the excited states of both donor and acceptor in PNC, which is likely due to the short length and conjugation of the bridge. This result is consistent with steady state and time-resolved fluorescence experiments on PNC (data not shown), which showed a very low fluorescence yield from PDI in PNC compared to PNR and PNF. All TA features in PNC decayed to zero with a lifetime of 200 ps, indicating that the short distance and 
conjugation of the PNC bridge leads to rapid dissipation of excited state energy and rapid recombination of any charge-separated species.
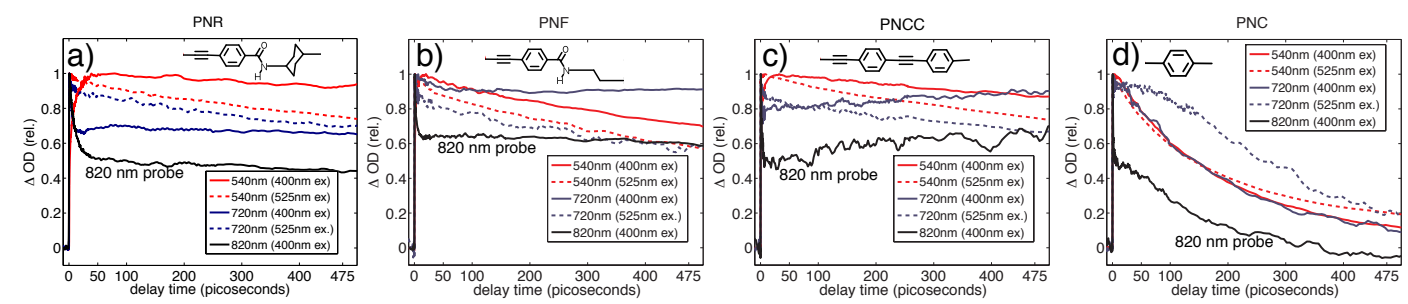

Fig. 2 Transient absorption traces of the four molecules. a) TA of PNR, indicating formation long-lived state involving 6T and PDI following excitation of 6T, but not of PDI. The absorption at $720 \mathrm{~nm}$ and $820 \mathrm{~nm}$ at long times is an indicator of the perylene anion in the charge-separated state [7]. b) TA of PNF, indicating long-lived state following excitation of 6T that involves 6T but not PDI. c) TA of

PNCC, indicating longer timescales of charge separation and recombination than PNF following excitation of 6T, but not PDI. d) TA of PNC, indicating charge separation following excitation of both $6 \mathrm{~T}(400 \mathrm{~nm})$ and PDI $(525 \mathrm{~nm})$, followed by recombination with a time scale of $200 \mathrm{ps}$.

\section{Conclusion}

Femtosecond TA spectroscopy has been conducted on molecular PN junctions consisting of a 6T oligomer as electron donor and a perylene bisimide (PDI)-napthalene bisimide (NDI) dimer as electron acceptor. In PNC, we observed charge separation from excited states of both donor and acceptor; in PNCC, PNF and PNR, which have longer bridges of varying flexibility and conjugation, we only observed charge separation from the donor. Recombination of the charge separated state occurs more rapidly in molecules with a short, conjugated bridge than in the other molecules with longer, flexible bridges, making the extraction of charges more difficult. Overall, a rigid bridge appears to be preferable to a flexible bridge for reducing the rate of charge recombination, and a short bridge appears to be necessary for charge separation to occur from both donor and acceptor.

\section{Acknowledgements}

We thank Dr. Ying-Zhong Ma and Dr. Matthew Graham for assistance with the experimental apparatus. This work was funded by the Helios Solar Energy Research Center, which is supported by the Director, Office of Science, Office of Basic Energy Sciences of the U.S. Department of Energy under Contract No. DE-AC02-05CH11231.

\section{References}

1. N. Cai, S.J. Moon, L. Cevey-Ha, T. Moehl, R. Humphrey-Baker, P. Wang, S. M. Zakeeruddin, and M. Gratzel. Nano Lett., 11, 1452 (2011).

2. J.Roncali. Acc. Chem. Res., 42, 1719 (2009).

3. J. M. Szarko, J. Guo, B.S. Rolczynski, and L.X. Chen. Nano Reviews, 2, 1 (2011).

4. L. X. Chen, S. Xiao, and L. Yu. J. Phys. Chem. B, 110, 11730 (2006).

5. J. Guo, Y. Liang, S. Xiao, J. M. Szarko, M. Sprung, M. K. Mukhopadhyay, J. Wang, L. Yu, and L. X. Chen. New J. Chem., 33, 1497 (2009).

6. M. Fujitsuka, K. Harada, A. Sugimoto, and T. Majima. J. Phys. Chem. A, 112, 10193 (2008).

7. D. Gosztola, M.P. Niemczyk, W. Svec, A. S. Lukas, and M. R. Wasielewski. J. Phys. Chem. A, 104, 6545 (2000).

8. D. Grebner, M. Helbig, and S. Rentsch. J. Phys. Chem., 99, 16991 (1995).

9. V. Wintgens, P. Valat, and F. Garnier. J. Phys. Chem., 98, 228 (1994) 